\title{
6 ERWACHSENENBILDUNG FÜR GEFLÜCHTETE
}

$\mathrm{Zu}$ Beginn dieses Kapitels soll noch einmal kurz auf das bereits beschriebene Modell zur Integration von Geflüchteten und Migrant/inn/en von AgER und STRANG (2008) eingegangen werden. Davon ausgehend wird die spezifische Situation von erwachsenen Asylwerber/inne/n und Asylberechtigten im Bildungssektor in Österreich behandelt. An dieser Stelle sind vor allem die folgenden Kerndomänen von Integration von besonderer Relevanz: Einerseits die „facilitators“ „language and cultural knowledge“ sowie „safety and stability“ und andererseits ,education“ als wichtiger Aspekt der Kategorie ,markers and means“.

Die genannten „facilitators“ sind für die Verwirklichungschancen von Geflüchteten in Österreich insofern wichtig, da sie Integration erleichtern oder behindern können. Die Aneignung von Sprache und das Wissen um die Abläufe, Erwartungshaltungen in Interaktionen sowie Informationen über das Leben in Österreich benötigt eine gewisse Zeit, die hierfür auch gewährt werden sollte. Der Bereich „cultural knowledge“ kann hier nicht detaillierter diskutiert werden, weil damit eine große Anzahl an weiteren Themenfeldern verbunden ist, die den Rahmen dieser Arbeit sprengen würden. Analog zur Aneignung von Sprachkenntnissen ist aber festzuhalten, dass formelle Kursmaßnahmen wie die Werte- und Orientierungskurse des Österreichischen Integrationsfonds nicht ausreichen. Zusätzlich müssen Möglichkeiten geschaffen werden, um einen alltäglichen Austausch über Vorstellungen eines gelingenden Zusammenlebens zu ermöglichen. Hierbei spielen Deutschkenntnisse erneut eine wichtige Rolle.

Nachdem viele Asylwerber/innen und Asylberechtigte aus bedrohlichen Situationen aufgebrochen und teilweise über sehr lange Zeiträume unterwegs waren, ist nach der vorläufigen Ankunft vor allem das Bedürfnis nach Sicherheit sehr groß (AGER \& Strang 2008, 183). Dies wurde in Gesprächen über die persönliche Situation in Österreich ebenfalls immer wieder betont (vgl. Interview ASYLWERBER IRAN ST. ANDRÄWöRDERn 2018). Was den Aspekt der Stabilität betrifft, sind zwei wesentliche Faktoren relevant: Einerseits ist für die Dauer des Asylverfahrens offen, ob Geflüchtete überhaupt in Österreich bleiben können, was große Unsicherheit auslöst. Auf der anderen Seite können bereits aufgebaute Beziehungen zu Unterstützer/inne/n unerwartet unterbrochen werden, wenn beispielsweise Quartiere geschlossen und die Bewohner/innen anderswo untergebracht werden (vgl. Ager \& Strang 2008, p. 184). Dies konnte in St. AndräWördern nach der vorläufigen Schließung des ÖJAB-Hauses Ende Juni 2018 beobachtet werden. Auch für die Integration von Geflüchteten im Bildungssektor zeigt sich also, dass

„,...] solange Grundbedürfnisse nach geeignetem Wohnraum, gesundheitlicher und psychischer Stabilität (Traumatisierungen, Foltererlebnisse, Zurücklassen von Familienmitgliedern), Ausbildung/Berufsausbildung und finanzieller Absicherung nicht gegeben sind, [...] Integration massiv erschwert [wird]. “ (KöGLER 2008, p. 179) 
In diesem Kapitel wird eine detaillierte Auseinandersetzung mit entscheidenden Maßnahmen zur Integration von erwachsenen Geflüchteten im Bildungssektor vorgenommen. So werden die wichtigsten thematischen Angebote, nämlich Deutsch-, Basisbildungs- und Pflichtschulabschlusskurse, anhand von empirischen Beispielen behandelt. Davon ausgehend wird sodann näher auf die spezifische Situation insbesondere von Asylwerber/inne/n eingegangen, welche für deren Verwirklichungschancen von großer Bedeutung ist. Anschließend folgt die Beschäftigung mit den wichtigsten individuellen und strukturellen Faktoren, die für die Integration von Geflüchteten im Bildungssektor relevant sind. Abschließend werden die zentralen Erkenntnisse dieses Kapitels nochmals zusammengefasst.

\subsection{Deutschkurse für Asylwerber/innen und Asylberechtigte}

Deutschkenntnissen wird für die Integration von Geflüchteten in Österreich eine entscheidende Bedeutung zugeschrieben (GATT 2014, p. 163). Stets wird die Beherrschung der deutschen Sprache als zentrale Voraussetzung für Integration und Zugehörigkeit hervorgehoben (vgl. HeInEMANN 2017). Wenn zusätzlich davon auszugehen ist, dass ein gewisses Deutschniveau durch Sprachkurse erreicht werden kann, wird verständlich, warum Deutschkurse von staatlicher Seite als eine der wichtigsten Integrationsmaßnahmen angesehen werden. Allerdings blieb die Verfügbarkeit von qualitativ hochwertigen Angeboten in den Jahren der großen Fluchtbewegungen von 2015 und 2016 in den meisten Bundesländern deutlich hinter der faktischen Nachfrage zurück (SOS Mitmensch 2017). Vor allem Asylwerber/innen können eigentlich nur kostenlose Angebote nutzen, wobei außerdem der Anfahrtsweg keine zusätzlichen Kosten verursachen darf, da diese mit den monatlichen 40 Euro „Taschengeld“ aus der Grundversorgung kaum abzudecken sind. ${ }^{19}$ Insbesondere durch die große Zahl an Geflüchteten, die in den Jahren 2015 und 2016 nach Österreich kamen, entstanden vielerorts ehrenamtliche Initiativen, um diese Menschen zu unterstützen. Neben vielen Soforthilfemaßnahmen wurden in einigen Orten auch kostenlose Deutschkurse angeboten oder individuell Geflüchtete beim Erlernen der deutschen Sprache unterstützt. Dies war auch in jenen Gemeinden der Fall, in denen die Feldforschungen als Basis dieser Arbeit durchgeführt wurden. Die Beschreibung der Deutschkurse in der Gemeinde St. AndräWördern und im Mostviertel sowie aller weiteren Bildungsmaßnahmen, die in diesem Kapitel erwähnt werden, basiert auf dem Stand zum Zeitpunkt der Datenerhebung im Jahr 2018. Inwieweit sich die Situation seither verändert hat wurde von den Autor/inn/ en nicht erhoben.

19 Die Grundversorgung für Asylwerber/innen während des Asylverfahrens umfasst Verpflegung, Unterkunft und andere Unterstützungsleistungen (ASYLKOORDINATION ÖSTERREICH 2018). Es besteht entweder die Möglichkeit in organisierten Quartieren oder in privaten Unterkünften zu wohnen. Je nach Art der Unterbringung sind damit auch unterschiedliche Leistungen aus der Grundversorgung verbunden. So erhalten Asylwerber/innen in organisierten Quartieren pro Monat 40 Euro „Taschengeld“ sowie Vollverpflegung beziehungsweise Verpflegungsgeld für jene Mahlzeiten, die nicht vom Quartier bereitgestellt werden (ebd.). 


\subsubsection{Empirische Beispiele aus St. Andrä-Wördern und dem Mostviertel}

Im Folgenden werden einige Anbieter von Deutschkursen in den Erhebungsregionen vorgestellt und deren spezifische Zugänge behandelt.

\section{Deutschkurs von Ehrenamtlichen in der Gemeinde St. Andrä-Wördern}

Mit der Unterbringung von weiteren Asylwerber/inne/n in St. Andrä-Wördern wurde zunächst parallel zum ÖJAB-Haus von Mitgliedern von „St. Andrä-Wördern hilft" ein zusätzlicher Deutschkurs angeboten. In den ersten Wochen wurde in der Großgruppe und mithilfe eines Übersetzers für Arabisch im Keller des Gemeindeamtes unterrichtet. Danach wurden die Maßnahmen zusammengefasst, sodass in der Bücherei dreimal pro Woche circa 10 bis 20 Personen unterrichtet wurden. Die Kursniveaus reichten dabei von einer ersten Alphabetisierung über A1 und A2 bis zu B1 und einem einzelnen Schüler, der auf die B2-Prüfung vorbereitet wurde (vgl. Interview

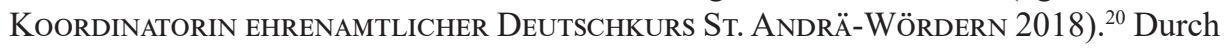
diese Aufteilung in Kleingruppen wurde ein persönlicher und unmittelbarer Unterricht sichergestellt, von dem die Teilnehmer/innen der Kurse profitierten. Dies brachte aber die Herausforderung mit sich, für jeden Termin ausreichend Lehrpersonen zu organisieren, um diese intensive Betreuung sicherzustellen. Dabei stellte sich heraus, dass es förderlich ist, für eine größtmögliche Kontinuität zu sorgen, indem die Ehrenamtlichen längerfristig dieselben Schüler/innen unterrichteten (vgl. Interview EHRENAMTLICHE DEUTSCHLEHRERIN St. ANDRÄ-WÖRDERN 2018).

Inhaltlich wurde vor allem an den Kompetenzen Sprechen, Hören, Lesen und Schreiben gearbeitet, wobei je nach Situation unterschiedliche Schwerpunkte gesetzt wurden. Die Unterrichtsmaterialien waren einerseits Bücher, welche über den „Grenzenlos“ Solidarfonds finanziert und dann auch gebraucht wiederverwendet wurden. Einige der Bücher wurden von der Gemeinde finanziert; ein weiterer Antrag zum Ankauf von Deutschlehrbüchern wurde allerdings abgelehnt (Interview LEITERIN „ST. ANDRÄ-WÖRDERN HILFT“ 2018). Die Deutschlehrer/innen hatten aber andererseits die Möglichkeit, Unterlagen im Gemeindeamt kostenlos zu kopieren und erhielten die Räumlichkeiten in der Bücherei über die Gemeinde kostenfrei zur Verfügung gestellt. Die entsprechenden Deutschprüfungen nach dem europäischen Referenzrahmen für Sprachen (A0-C2) mussten von den Teilnehmer/inne/n des Deutschkurses dann extern in Wien belegt werden. Als Prüfungsgebühr wurden 145 Euro eingehoben, die die Schüler/innen entweder selbst, mit der Unterstützung des „Grenzenlos“ Solidarfonds oder anderer Spender/innen bezahlten. Die Prüfungsvorbereitung dazu fand im Bedarfsfall auch im Einzelunterricht statt, um größtmögliche Chancen zu bieten, die Prüfung zu bestehen.

Einer der zentralen Punkte, welcher von den Ehrenamtlichen in Gesprächen immer wieder betont wurde, war, dass es sich beim Deutschkurs nicht nur um eine 20 Die Koordinatorin des ehrenamtlichen Deutschkurses ist zusätzlich selbst als Deutschlehrerin tätig. 
Bildungsmaßnahme handelt. So erhielten die Geflüchteten über die Deutschlehrer/innen Unterstützung in unterschiedlichen Belangen, die von rechtlichen Fragen im Asylverfahren, Hilfe bei der Übersetzung von Briefen bis hin zum Austausch über den Alltag in Österreich reichten.

„,Wenn halt irgendwelche Sorgen sind, dann hören wir ihnen auch zu im Kurs, dann ist eine Stunde einmal nix. Also da ist einer einmal untergetaucht und man hat nicht gewusst, wo er ist und das haben sie mir lang und breit erklärt und erzählt und ich hab' gemerkt, sie brauchen das halt einfach. Und sie haben halt das Ganze auf Deutsch [erzählt], somit haben wir ein bisschen Deutsch gemacht. Aber das haben sie gebraucht. Also wir versuchen halt auch ein offenes Ohr zu haben, wenn sie das brauchen." (Interview KOORDINATORIN EHRENAMTLICHER DeutschKurs ST. ANDRÄ-WÖRDERn 2018)

Die Ausgestaltung des Deutschkurses stellte allerdings an die Lehrenden recht hohe Anforderungen. Dadurch, dass die Teilnehmer/innen auch kurzfristig einsteigen konnten, gab es immer wieder logistische Herausforderungen, was Materialien und Plätze anbelangte. Infolge der Niederschwelligkeit und des kostenlosen Angebots kam es wiederholt vor, dass Geflüchtete unentschuldigt dem Kurs fernblieben, sodass einzelne Lehrer/innen ihre Tätigkeit aus Frustration darüber einstellten (vgl. Interview EHRENAMTLICHe DeutschleHrerin ST. ANDrä-WÖRdERn 2018). Eine weitere Schwierigkeit stellten die sehr unterschiedlichen Wissensstände dar, auf die durch die Aufteilung in Kleingruppen nach Möglichkeit reagiert wurde. $\mathrm{Zu}$ den Vorteilen der ehrenamtlich organisierten und durchgeführten Kurse zählte, dass diese weitgehend unabhängig gestaltet werden konnten. Auch wenn die Lehrmaterialen Grundlagen für den Prüfungsstoff bildeten, konnte auf die Bedürfnisse der Gruppe recht unkompliziert eingegangen werden und somit die Art und Weise der Vermittlung der jeweiligen Alltagssituation und dem Bedarf angepasst werden.

\section{Deutschkurs von Ehrenamtlichen im Mostviertel}

In der beforschten Region im Mostviertel wurden seit 2015/16 in den Gemeinden A und C ebenfalls Deutschkurse von Ehrenamtlichen organisiert und durchgeführt. Als Best-Practice-Beispiel wird der ehrenamtliche Deutschkurs in Gemeinde A detaillierter vorgestellt: Um die in der Gemeinde vorhandene breite Unterstützungsbereitschaft besser koordinieren und verwalten zu können, wurde dafür 2015 ein eigener Verein gegründet. Zu den Unterstützungsleistungen zählten beispielsweise finanzielle Spenden, ebenso wie Sachspenden und ehrenamtliche Arbeit im Bereich der Sprachvermittlung oder der Kinderbetreuung. Auch hier wurden mehrere Gruppen mit ähnlichen Sprachniveaus gebildet. Als Unterrichtsmaterialen wurden überwiegend Materialien von der Homepage des Vereins „Deutsch.Fit“" verwendet. Diese wurden speziell für den ehrenamtlichen Deutschunterricht für Asylwerber/innen konzipiert und berücksichtigen deren spezifische Situation. Ein Merkmal ist beispielsweise die Strukturierung in kurze Lektionen, die dem Umstand Rechnung trägt, dass Asylwerber/innen oftmals Kurse 
auch wieder abbrechen müssen aber damit trotzdem das Gefühl erhalten, bestimmte Kapitel abgeschlossen zu haben (VEREIN DEUTSCH.FIT o.J.). Übergeordnetes Ziel der ehrenamtlichen Unterstützung im Rahmen des Willkommens-Vereins war die Hilfe zur Selbsthilfe. Dies spiegelte sich auch darin wider, dass Familien mit CD-Playern ausgestattet wurden, um das Selbststudium der deutschen Sprache außerhalb des Kurses zu unterstützen oder dass für engagierte Lernende mit einem gewissen Fortschritt nach Möglichkeit externe ÖSD-Prüfungen zur Erlangung von Sprachzertifikaten vom Verein finanziert wurden. Der Verein wurde im Laufe des Jahres 2018 wieder aufgelöst, da der Bedarf nicht mehr gegeben war. Der Großteil der ehemaligen Asylwerber/innen übersiedelte nach der Zuerkennung des Asylstatus in den urbanen Raum oder musste die Gemeinde aus anderen Gründen verlassen (Interview OBMANN WiLlKommens-Verein GEMEINDE A 2019).

\section{Deutschkurse der Firma MENTOR im Mostviertel}

Ein für ländliche Regionen interessantes Kurskonzept können dezentral organisierte Maßnahmen sein. Beispielhaft können hier die Deutschkurse der Firma MENTOR angeführt werden. In der beforschten Region im Mostviertel wurden in der ersten Jahreshälfte 2018 in der Gemeinde C Deutschkurse der Stufen A0 und A1 von einer zertifizierten DaF/DaZ-Trainerin täglich von Montag bis Freitag im Sitzungssaal der Gemeinde unterrichtet. Zielgruppe dieser Maßnahme, die aus Mitteln des Bundes und des Landes Niederösterreich finanziert wurde und durch Kooperationsarbeit des mobilen Flüchtlingsdienstes der Diakonie zustande kam, waren Asylwerber/innen mit hoher Anerkennungswahrscheinlichkeit. Dieses Kurskonzept war aus verschiedenen Gründen bemerkenswert, vor allem da es die spezifische Situation von Asylwerber/ inne/n berücksichtigte: 1) Die Mobilitätskosten zum Kursstandort konnten durch die dezentrale Organisation geringgehalten werden. Die Anfahrt für die Teilnehmenden im Einzugsgebiet wurde für die benötigten Streckenabschnitte mitfinanziert. 2) Die Lernenden wurden gezielt auf die ÖSD-Prüfung vorbereitet, die am Ende des Kurses abgelegt werden sollte. Auch hierbei wurden alle anfallenden Kosten vom Projekt übernommen. 3) Die Maßnahme fand fünfmal wöchentlich statt. Diese Regelmäßigkeit garantierte einen kontinuierlichen Lernfortschritt und wirkte sich auch positiv auf die Tagesstruktur der Kursteilnehmer/innen aus (Interview DAZ-TraINERIN MENTOR 2018; Interview SozIALARbeITERIN DiakONIE - MOBILER FlÜCHTLINGSDIENST 2018).

\subsubsection{Sprachvermittlung durch Kursmaßnahmen und in alltäglichen Interaktionen}

Trotz der beträchtlichen Bedeutung eines zugänglichen Angebotes an Deutschkursen stellt die Annahme, dass Sprachkenntnisse vor allem durch formalisierte Bildungsmaßnahmen verbessert werden, aus linguistischer Perspektive eine Illusion dar (vgl. Plutzar 2010, pp. 125 ff.). Sprache wird demnach vor allem durch Sprechen gelernt und damit in der Anwendung im Alltag und weniger in einer Kurssituation 
(ebd.). Zusätzlich sind Lerntypen sehr unterschiedlich und manche Menschen können sich aus den formellen Lernsituationen in Kursen nur wenig mitnehmen (BEOBACHTUNGSPROTOKOLL MUTTERSPRACHLICHE Interviews IN ST. ANDRÄ-WÖRDERN 2018). Andererseits gibt es viele andere Möglichkeiten, wie Sprachen gelernt werden können. So erzählte eine der ehrenamtlichen Deutschlehrer/innen, dass manche der Schüler/ innen über das Internet lernten, gemeinsam mit anderen Personen, die schon besser Deutsch konnten, oder in Einzelfällen selbstständig durch Bücher oder deutschsprachige Filme mit Untertiteln (Interview KOORDINATORIN EHRENAMTLICHER DEUTSCHKURS St. ANDRÄ-WÖRDERN 2018). Andere Geflüchtete benötigten allerdings ein Mehr an Struktur und profitierten stark von den angebotenen Kursen und eventuell damit verbundenen Hausaufgaben (ebd.). Insgesamt sind erfolgversprechende Lernstrategien sehr individuell und von der persönlichen Situation abhängig. In allen Fällen sind jedoch Möglichkeiten, das Gelernte auch im Alltag anzuwenden von zentraler Bedeutung. So wurde berichtet, dass vor allem Geflüchtete, die sich häufiger auf Deutsch mit anderen Menschen unterhielten, viel raschere Fortschritte erzielten (Interview EHRENAMTLICHE DEUTSCHLEHRERIN IN ST. ANDRÄ-WÖRDERN 2018):

„Das haben wir beim Familienvater sehr gut gesehen. Wie der beim SOMAMarkt [Sozialmarkt] ehrenamtlich gearbeitet hat, ist es mit dem Sprechen besser gewesen. Ganz einfach, ich mein' die Kunden, die Leute, die dort arbeiten, die reden nicht Farsi mit ihm, da kann er machen was er will. Das [war] wesentlich besser dann. “ (Interview LEITERIN „ST. ANDRÄ-WÖRDERN HILFT“ 2018)

Die Verbesserung der Sprachkenntnisse durch Interaktionen im Alltag führt dazu, dass sich diese Fortschritte auf Bereiche beziehen, die für die Geflüchteten von unmittelbarer Wichtigkeit sind, um sich in Österreich besser zurechtzufinden. Auch ein Verständnis für den österreichischen Dialekt, der sich in manchen Fällen doch stark von der Schriftsprache unterscheidet, kann in Sprachkursen nur sehr schwer vermittelt werden (vgl. Interview LeITER BACH BiLdungszentrum 2018). Es gab allerdings Versuche diesen sehr praxisorientierten Zugang mit dem Anspruch, zur selbstständigen Bewältigung des Alltags auf Deutsch zu befähigen, in Kursmaßnahmen umzusetzen:

„,Wir versuchen hier ein Deutsch den Leuten nahe zu bringen, das gesprochen wird. [...]. Natürlich in der verschriftlichten Form, weil, man braucht einen Anhaltspunkt, aber gesprochenes Deutsch, Alltagsdeutsch. Kein Zeitungsdeutsch, sondern Alltagsdeutsch, wie man es für den Alltag braucht, für das Einkaufen und so weiter - es ist eh schwierig genug. " (Interview DAZ-LERNBEGLEITER BASISBILDUNG „BILDUNG HAT WERT“ 2018)

Deutschkenntnisse werden im Diskurs in Österreich häufig mit erfolgreicher Integration gleichgesetzt (vgl. DE CILla \& DorostKar 2014). Dabei wird jedoch außer Acht gelassen, dass geringe Sprachkenntnisse nicht der einzige Grund für die gesellschaftliche Marginalisierung von Geflüchteten sind. Weitere relevante Faktoren in diesem Zusammenhang sind beispielsweise die rechtlichen Rahmenbedingungen, soziale Herkunft und strukturelle Diskriminierung in Österreich (vgl. Plutzar 2010, 
p. 127). Deutschkenntnisse können dabei nicht nur als Voraussetzung für gelingende Integration angesehen werden, sondern stellen für sich schon einen wichtigen Integrationserfolg dar (ebd.). Außerdem wird tendenziell davon ausgegangen, dass Deutsch als Umgangssprache im politischen Diskurs in Österreich ein konstituierendes Element der kollektiven Identität darstellt (DE CiLla \& DorostKar 2014, pp. 150 ff.). Dadurch werden Menschen, welche die Sprache nur in geringem Ausmaß beherrschen, automatisch exkludiert. Dieser „monolinguale Habitus“ (Gogolin 2008 [1994]) führt dazu, dass mangelnde Deutschkenntnisse von Asylwerber/inne/n und Asylberechtigten als Defizit wahrgenommen werden, ohne dass anerkannt wird, dass diese Menschen vielleicht schon mehrere andere Sprachen beherrschen. Die Verantwortung bezüglich der Aneignung von Deutschkenntnissen wird analog zur Debatte über Assimilation (vgl. Abschnitt 2.2) häufig einseitig den Geflüchteten zugeschrieben (GATT 2014, p. 168), ohne deren Situation und Möglichkeiten entsprechend zu reflektieren. Das Angebot von Deutschkursen, die auf die Situation von Asylwerber/inne/n und Asylberechtigten in Österreich eingehen, ist daher von besonderer Bedeutung. Deutschkenntnisse können zu mehr Selbstständigkeit im Alltag, im Umgang mit Behörden oder beim Einkaufen beitragen und eröffnen neue Möglichkeiten auf dem Arbeitsmarkt. Gerade auch im Kontakt mit anderen Menschen zeigt sich die Wichtigkeit von Deutschkenntnissen, deren politische Instrumentalisierung (PLUTZAR 2010, p. 137) sollte allerdings kritisch hinterfragt werden.

\subsection{Integration von Asylwerber/inne/n im Bildungssektor}

Bildung stellt in Österreich eine wichtige Komponente für die Verbesserung von Verwirklichungschancen von Geflüchteten dar und wird in unterschiedlichen Integrationsmodellen an prominenter Stelle genannt (AgER \& Strang 2008; Heckmann 2003). Auch in den Interviews wurde der Stellenwert von Bildung sehr hoch eingeschätzt:

„Bildung ist alles würd' ich sagen: Erstens die Sprache zu sprechen, Schilder lesen zu können, in der Lage zu sein ein Formular auszufüllen [...] ist das A und $O$. Also ja, ich würd' sagen, es ist einfach alles und das Allerwichtigste. " (Interview Trainerin PflichtschulabschlussKurs PROSA 2018)

Insbesondere im Zusammenhang mit der Partizipation von Geflüchteten an der österreichischen Gesellschaft spielt Bildung eine wichtige Rolle (vgl. Interview LEITER BACH Bildungszentrum 2018). Sie ist sowohl eine bedeutende Errungenschaft als auch eine Möglichkeit zur Verbesserung von Integration im Sinne von Verwirklichungschancen. An dieser Stelle ist es wichtig zu betonen, dass damit nicht ausschließlich die Vermittlung von fachlichem Wissen gemeint ist. Im weiteren Sinne inkludiert Bildung die Befähigung zum kritischen Denken, außerdem erweitert sie den eigenen Horizont und fördert einen differenzierten Umgang mit Informationen (vgl. Interview DiaKoN Pfarre St. Andrä vor dem Hagental 2018). Auf den ,,persönlichkeitsbildenden Wert 
und emanzipatorischen Charakter" von Bildung wird unter anderem von JENEWEIN (2014, p. 45) hingewiesen.

Ein derart weiter gefasstes Verständnis von Bildung beschränkt Förderung in diesem Bereich jedoch keineswegs auf Geflüchtete oder Personen mit Migrationserfahrung, sondern ist als gesamtgesellschaftlich erstrebenswert anzusehen. Trotzdem ist vor allem die Situation von Asylwerber/inne/n und Asylberechtigten eine spezifische: Neben dem Erwerb von fachlichen Kenntnissen in unterschiedlichen Bereichen ist gleichzeitig die Herausforderung in einem neuen sozialen und kulturellen Umfeld anzukommen zu bewältigen. In diesem Zusammenhang spielen Bildung, aber auch in besonderer Weise Sprachkenntnisse, eine zentrale Rolle:

„,...] sagen wir, sie kriegen eine Sprache, also sie können mitsprechen. Ja, sie kriegen Möglichkeiten zu verstehen und von sich zu geben, sonst sind sie ja stumm, verstehen nicht und können nicht mitreden. Ich glaub' das ist wichtig mal am Anfang, die Möglichkeiten zu geben und dann sie [zu] unterstützen. " (Interview LEITER BACH BiLdungszenTRum 2018)

Es kann an dieser Stelle nicht genauer auf die unterschiedlichen Möglichkeiten für Geflüchtete auf dem Bildungssektor in Österreich eingegangen werden. In den Interviews wurde allerdings immer wieder betont, dass viele Asylwerber/innen und Asylberechtigte nicht in ausreichendem Umfang über das Bildungssystem in Österreich informiert waren. (vgl. Interview KoORDINATORIN EHRENAMTLICHER DEUTSCHKURS ST. ANDRÄ-WÖRDERn 2018). Es gab und gibt zwar diesbezügliche Infomaterialen und Onlineinformationen in jenen Sprachen, die häufig von Geflüchteten gesprochen werden, allerdings gelangen diese oft nicht an die Personen, welche sie benötigen würden (ebd.). Auskünfte über das Bildungssystem in Österreich werden jedoch häufig von ehrenamtlichen Unterstützer/inne/n weitergegeben, deren diesbezügliche Kontakte eine wichtige Rolle spielen. Ein weiterer Ansatz hierzu sind organisierte Angebote wie beispielsweise die muttersprachliche Bildungs- und Berufsberatung des Bildungszentrums BACH in Mödling. ${ }^{21}$ Dort können sich Geflüchtete und Migrant/inn/en einzeln oder in Gruppen in ihrer Muttersprache beraten lassen. Es wird auf diese Weise versucht, die Personen individuell auf ihrem weiteren Bildungsweg zu unterstützen. Dabei werden vor allem Informationen zum Bildungssystem in Österreich weitergegeben, aber auch mögliche Förderungen und Schulungen zum Berufseinstieg besprochen. Ebenfalls wichtig ist es dabei Erwartungen abzuklären und über die Voraussetzungen und Dauer von Ausbildungen zu informieren, welche zur Ausübung des gewünschten Berufes befähigen.

Im Folgenden wird exemplarisch auf Basisbildungskurse und Pflichtschulabschlusskurse für Geflüchtete näher eingegangen. Dabei sollen einerseits die Rahmenbedingungen der Bildungsmaßnahmen für diese Zielgruppe näher erläutert und andererseits Herausforderungen aber auch Strategien des Umgangs damit aufgezeigt werden. Die meisten dieser Angebote waren nicht direkt in jenen Gemeinden lokalisiert, auf denen

21 Genauere Informationen zum BACH Bildungszentrum in Mödling werden in Abschnitt 6.2.2 genannt. 
der Schwerpunkt der empirischen Erhebungen für diese Publikation lag. Daher wurden Beispiele ausgewählt, die entweder einen regionalen Bezug haben, also beispielsweise von Asylwerber/inne/n und Asylberechtigten aus den Gemeinden besucht wurden, oder die in besonderer Weise eine thematische Relevanz aufweisen.

\subsubsection{Basisbildung}

Basisbildungskurse für Erwachsene dienen in erster Linie als Unterstützung zum (Wieder-)Einstieg in das österreichische Bildungssystem und richten sich an Personen, bei denen ein grundlegendender Bildungsbedarf besteht. Sie sollen die Teilnehmer/ innen dazu befähigen, erste wichtige Schritte im Rahmen ihrer Bildungslaufbahn zu setzen und sie für weitere Maßnahmen, wie etwa Pflichtschulabschlusskurse qualifizieren (vgl. EntwicklungSPARTNERSChaft „Mehrsprachig Vielfältig“ 2014, p. 8). Wichtig ist hierbei vor allem ein praxisorientierter und niederschwelliger Zugang, der auf die Lernvoraussetzungen und speziellen Bedürfnisse der Lernenden eingeht (ebd.). Inhaltlich ist eine breitere Palette als nur die Vermittlung von Deutschkenntnissen inkludiert. So beinhalten Basisbildungskurse zumeist Sprachen, Mathematik sowie Informations- und Kommunikationstechnologien als thematische Schwerpunkte

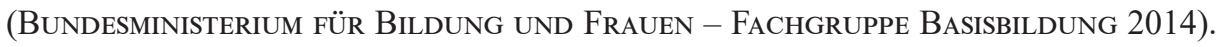
Hinsichtlich der Organisation dieser Bildungsmaßnahmen manifestierten sich jedoch bedeutende Unterschiede zwischen verschiedenen Anbietern. Beim Basisbildungskurs des Roten Kreuzes in Tulln wurde eine Gruppe von Teilnehmer/inne/n in allen Gegenständen gemeinsam unterrichtet. Im Falle von „Bildung hat Wert“ im Mostviertel gab es hingegen verschiedene Kurse, die jeweils einen einzelnen Fachbereich abdeckten. In beiden Fällen erhielten die Lernenden eine Teilnahmebestätigung, die als Nachweis über die erfolgreiche Absolvierung des Kurses vorgelegt werden konnte. Entsprechend der grundlegenden Ausrichtung von Basisbildungsangeboten bestehen also verschiedene Möglichkeiten zur Umsetzung dieser Angebote.

Die ersten Basisbildungskurse für Erwachsene entstanden in Österreich Ende der 1980er und Anfang der 1990er Jahre in Wien (KASTNER 2016, p. 18). Prinzipiell wurden dafür die folgenden Zielgruppen festgelegt: Menschen mit Deutsch als Muttersprache, aber entweder ohne positivem Pflichtschulabschluss oder mit dennoch vorhandenem Bildungsbedarf sowie Personen mit Migrations- oder Fluchterfahrungen und zusätzlichen Bildungsbedürfnissen (ebd., p. 42). Dabei ist anzumerken, dass, insbesondere in der Gruppe der Asylwerber/innen und Asylberechtigten, eine beträchtliche Heterogenität in Bezug auf die Wissensstände in einzelnen Fachgebieten besteht. Auf diesen Umstand wird in Abschnitt 6.3.1 noch näher eingegangen werden. Basisbildungsangebote richten sich also an bestimmte Zielgruppen, deren Benennung eine wichtige Rolle spielt. Kastner (2016, p. 3) schlägt in diesem Zusammenhang die Bezeichnung „bildungsbenachteiligte Erwachsene mit Basisbildungsbedarf" vor. Der etwas sperrige Begriff deutet darauf hin, dass ,behindernde und verhindernde Strukturen und Mechanismen am Werke waren/sind, welche die Bildungschancen und Lernvoraussetzungen negativ 
beeinflusst haben und weiter beeinflussen“ (ebd.). Damit wird darauf hingewiesen, dass Bildungsbedarf von Erwachsenen eher durch gesellschaftliche Ungleichheit entsteht und daher kein individuelles Problem ist, sondern vorwiegend strukturelle Ursachen hat (vgl. ebd., p. 9).

Neben den inhaltlichen Schwerpunkten wird in Basisbildungskursen auch „Lernen lernen“ als Querschnittsmaterie behandelt (STEINER, EGGER-STEINER \& BAUMEGGER 2018). Durch Anleitung zum Zeitmanagement und zur Entwicklung von Lernstrategien sollen die Kompetenzen der Teilnehmer/innen zur eigenständigen Wissensaneignung und persönlichen Weiterentwicklung erhöht werden. Darüber hinaus bieten formelle Kursangebote Gelegenheiten soziale und kulturelle Erwartungen an Geflüchtete in Österreich zu thematisieren. Die Bedeutung von Höflichkeit und Pünktlichkeit beispielsweise kann unterschwellig im Umgang miteinander in den Basisbildungsmaßnahmen betont werden (ebd.). Durch diesen Zugang können entsprechende Angebote einen Beitrag dazu leisten, dass Geflüchtete in der österreichischen Gesellschaft ankommen können und sich schneller selbstständig zurechtfinden.

\section{Basisbildungskurs des Roten Kreuzes in Tulln}

Das Rote Kreuz in Tulln bot mit Stand Juni 2018 zwei Basisbildungskurse an, welche Großteils aus Spendengeldern finanziert wurden ${ }^{22}$. Diese Kurse wurden für die Teilnehmer/innen kostenlos in zwei Gruppen zu je 15 Personen geführt. Die Mehrheit waren Asylwerber/innen unter 30 Jahren aus unterschiedlichen Herkunftsländern. Vereinzelt nahmen an den Kursen auch Asylberechtigte teil, denen seitens des AMS keine Maßnahme zugeteilt wurde oder die eine längere Unterbrechung zwischen anderen Bildungsangeboten zu überbrücken hatten. Die Kurse des Roten Kreuzes wurden dabei nicht nur von Geflüchteten aus Tulln besucht, auch aus den umliegenden Ortschaften nahmen Asylwerber/innen und Asylberechtigte teil. Die Fahrkosten für die An- und Abreise mit öffentlichen Verkehrsmitteln wurden vom Kursträger übernommen, obwohl dies eine erhebliche finanzielle Belastung darstellte und wegen mangelnder Ressourcen die Kursdauer verringert werden musste.

Jeweils zwei Trainer/innen unterrichteten die Gruppen im Umfang von 15 Stunden pro Woche in den Fächern Deutsch, Mathematik, Englisch und Informatik. Dabei gab es keine unterschiedlichen Leistungsgruppen, es wurden alle Teilnehmer/innen gleichzeitig unterrichtet. Dadurch, dass der Unterricht von zwei Personen geleitet wurde, bestand jedoch die Möglichkeit, dass der Schwierigkeitsgrad der Aufgaben individuell an das Können angepasst werden konnte. Der Vorteil dieses Formats lag darin, dass schwächere Kursbesucher/innen von jenen lernen konnten, die bereits über mehr Wissen im jewei-

22 Vom Roten Kreuz in Tulln wurden außerdem die Integrationsprojekte „X-Change“ und „Cross-Talk“ durchgeführt und Unterstützung bei der Suche nach vermissten Familienmitgliedern beziehungsweise bei der Familienzusammenführung bereitgestellt. Bei den oben genannten Projekten ging es vor allem darum, in Schulen oder mit Erwachsenengruppen Begegnungsmöglichkeiten zwischen Schüler/inne/n und Geflüchteten zu schaffen, um aus erster Hand einen Austausch über die jeweiligen Lebensrealitäten zu ermöglichen. 
ligen Bereich verfügten. Das konnten in den verschiedenen Gegenständen unterschiedliche Personen sein, sodass beispielsweise für jene, die in Mathematik Schwierigkeiten hatten, Erfolgserlebnisse möglich waren, wenn sie Kolleg/inn/en in Deutsch weiterhelfen konnten (vgl. Interview MitaRBEITERIN MigRATIONSABTEILUNG UND TRAINERIN Basisbildung Rotes Kreuz Tulln 2018). Es sollte allerdings darauf geachtet werden, dass Geflüchtete weder über- noch unterfordert werden. Laut eigener Aussage war das durch unterschiedliche Schwierigkeitsgrade in den Aufgabenstellungen und das Unterrichten durch zwei Trainer/inne/n durchaus zu bewerkstelligen (vgl. ebd.).

Wichtig für den Basisbildungskurs war und ist, dass die Lerninhalte an die Bedürfnisse der Lernenden angepasst werden. So wurden Informationen zum Versicherungssystem in Österreich oder das Ausfüllen eines Erlagscheines ebenfalls in den Unterricht einbezogen. Da das Rote Kreuz in Tulln kein zertifizierter Kursträger war, erhielten die Absolvent/inn/en nach dem positiven Abschluss des Kurses kein Zeugnis, sondern eine Teilnahmebestätigung. Die vermittelten Inhalte konnten aber genutzt werden, um die Integrationsprüfung des ÖIF abzulegen, welche aus Sprachkenntnissen auf A2-Niveau und einem Werteteil besteht und deren Nachweis für die Erfüllung der Integrationsvereinbarung notwendig ist.

\section{Basisbildungskurse als ganzheitliche Integrationsmaßnahme}

Wie oben erwähnt, stellen Basisbildungskurse für viele Teilnehmer/innen den Einstieg in das österreichische Bildungssystem dar. Insbesondere Geflüchteten, welche in den Herkunftsländern gar nicht oder nur für kurze Zeit Schulen oder andere Bildungseinrichtungen besucht haben, werden hier Grundkenntnisse zur Aneignung von Wissen vermittelt. So berichtete beispielsweise eine ehrenamtliche Deutschlehrerin aus St. Andrä-Wördern, dass im Deutschkurs manche Personen das erste Mal in ihrem Leben vor der Aufgabe standen ein Heft zu führen (vgl. Interview EhrenamtLiche DeutschLEHRERIN ST. ANDRÄ-WÖrdERn 2018). In diesem Sinne ging es in den Basisbildungskursen, aber auch in Sprachkursen, für viele Geflüchtete nicht zuletzt darum, formalisiertes Lernen, wie es im österreichischen Bildungssystem gefordert wird, zu erlernen:

„Menschen, die nie schreiben und lesen gelernt haben, die nicht in der Schule waren, für die ist Lernen sowieso etwas Neues. Der Zugang hier ist sehr, sehr individuell. Das bedeutet, auch wenn es Gruppen sind, dass wir trotzdem immer vielen persönlichen Dingen Raum geben und dass wir auch immer achten, wenn jemand langsamer ist oder mehr braucht. Dass man da wirklich darauf schaut. “ (Interview DaZ-Lernbegleiter BASISBILdung „BILdung HAT WerT“ 2018)

Damit stand also nicht unbedingt die reine Vermittlung von Wissen im Vordergrund, sondern es gab einen breiten Rahmen innerhalb dessen Kompetenzen vermittelt wurden, die zur eigenmächtigen Bestreitung des Alltags in Österreich wichtig sind. Interessant war dabei die Rolle, welche der Muttersprache der Teilnehmer/innen im Unterricht zukam. In Interviews wurde betont, dass diese dazu genutzt werden konnte, 
um Unklarheiten zu beseitigen und auf diese Weise Menschen mit geringeren Deutschkenntnissen zu unterstützen:

„,Wir stellen auch fest, dass es immer wieder Leute gibt, die in gewissen Gegenständen sehr gut sind - Mathematik [zum Beispiel]. Die helfen dann anderen [...]. Also, das stellen wir fest, wenn die Gruppendynamik gut ist, dass wir untereinander die Teilnehmer helfend auch einfach wahrnehmen. Und das ist oft sehr gut am Anfang, weil sie natürlich dann in Mathematik in ihrer eigenen Sprache das erklären können. " (Interview LEHRERIN PFLICHTSCHULABSCHLUSSKURS WIFI 2018)

Schließlich stellen Maßnahmen wie Deutsch-, Basisbildungs- und Pflichtschulabschlusskurse für Asylwerber/innen auch eine wichtige Möglichkeit dar, den eigenen Tagesablauf zu strukturieren. Das ist insofern wichtig, da viele während des Asylverfahrens zur Untätigkeit gezwungen werden, da kein Zugang zum regulären Arbeitsmarkt besteht.

„Für die Teilnehmer ist das Bedürfnis schon allein eine Tagesstruktur zu haben wahnsinnig groß. [...] Je näher das Ende vom Kurs kommt, da sind die Teilnehmer einfach gestresst, dass keine Maßnahme mehr für sie da ist. [Es gibt] mehrere Anfragen, wie es ausschaut. Ob sie weitergehen können, weil sie sonst keinen anderen Kurs haben und sie wollen aber nicht den ganzen Tag zu Hause sitzen und nichts machen. " (Interview Mitarbeiterin MigrationsabteILUng Rotes Kreuz TULLN 2018)

Im Gegensatz zu Freizeitaktivitäten, für die in den meisten Fällen die finanziellen Möglichkeiten stark eingeschränkt sind, ist bei Bildungsmaßnahmen eine höhere Verbindlichkeit gegeben. Erfahrungsgemäß sind viele Asylwerber/innen aus unterschiedlichen Gründen motiviert, an Sprach- oder Basisbildungskursen teilzunehmen:

„Ich habe gemerkt, da gibt es verschiedene Beweggründe, warum man einen Deutschkurs besucht. Es ist nicht immer nur jetzt wirklich das Deutschlernen. Für viele ist es oft Verschiedenstes: Tagesstruktur, vielleicht auch einmal in der Woche wo zu sein, aber auch für viele natürlich, weil es für das Asylverfahren wichtig ist. Das ist eine Info, das ist bei uns bekannt und die Teilnehmenden selber outen sich auch so. Also sagen sie wirklich, sie brauchen das für ihr Asylverfahren. Damit sie sagen können, sie machen was. " (Interview DAZ-LERNBEGLEITER BASISBILDUNG „BILDUng HAT WeRT“ 2018)

Unter den eben geschilderten Umständen und der schwierigen Situation von Geflüchteten in Österreich ist es allerdings für diese nicht leicht, die notwendige Energie aufzubringen, um sich wirklich auf Fortschritte im Bereich von Bildungsmaßnahmen oder Sprachkursen zu konzentrieren. Auf diesen Aspekt wird im Abschnitt 6.3.2 noch genauer eingegangen. 


\subsubsection{Pflichtschulabschlusskurse}

Der Pflichtschulabschluss ist in Österreich die Voraussetzung zur Zulassung an Berufsbildenden mittleren Schulen (Fachschule, Handelsschule) sowie Allgemeinbildenden und Berufsbildenden höheren Schulen (AHS, HTL, HAK) und wirkt sich auch bei der Suche nach einer Lehrstelle positiv aus (vgl. BRÜCKNER et al. 2017). Während Basisbildungskurse für viele Menschen einen niederschwelligen Einstieg in das Bildungssystem in Österreich darstellen, werden in den Kursen zum Pflichtschulabschluss bereits weiterführende fachliche Inhalte vermittelt. Dementsprechend verschiebt sich die Prioritätensetzung hin zu einer stärkeren Orientierung auf den Leistungsnachweis und die damit verbundenen Prüfungen. In der Erwachsenenbildung sind Pflichtschulabschlusskurse somit ein wichtiger Schritt in der Bildungskarriere von Menschen mit grundlegendem Bildungsbedarf. Um den Übergang aus Basisbildungskursen zu erleichtern, werden sogenannte Brückenkurse angeboten. Darin sollen die behandelten Inhalte gefestigt und die Teilnehmer/innen auf die weiteren Anforderungen vorbreitet werden (vgl. BRÜCKNER et al. 2017). Wichtig ist dabei, dass nicht versucht wird, die schulischen Strukturen und Organisationsformen eins zu eins auf Erwachsene anzuwenden, sondern dem Umstand einer umfassenderen persönlichen Erfahrung und der anderen Lebenssituation der Lernenden Rechnung zu tragen (ebd.). Außerdem dient Erwachsenenbildung auch dazu, die emanzipatorische Wirkung von Bildung im Sinne einer Verbesserung der Chancengleichheit durch lebenslanges Lernens zu entfalten (ebd.). Demnach sollen nicht nur Wissensinhalte vermittelt werden, sondern zusätzlich weitergehende Kompetenzen, die der nachfolgenden erfolgreichen Eingliederung in den Arbeitsmarkt dienen können. Dabei soll jedoch stärker als in der Schule auf den bereits vorhandenen Fähigkeiten der Erwachsenen aufgebaut werden, die sie im Laufe ihres Lebens in unterschiedlichen Bereichen bereits ausgebildet haben (HACKL-SCHUBERTH 2014, pp. 119 f.).

Um diesen Besonderheiten des Pflichtschulabschlusses für Erwachsene gerecht zu werden, sind auch die Unterrichtsgegenstände in den Kursen anders konzipiert als dies in der Schule der Fall ist. Seit 2012 wurden die bis dahin einzelnen Fächer für die Erwachsenenkurse als „transdisziplinäre Fächerkombinationen“ umgesetzt (BunDESMINISTERIUM FÜR UnTERRICht, Kunst Und Kultur 2013, p. 7). Das Curriculum sieht somit die folgenden Gegenstände vor: „Deutsch - Kommunikation und Gesellschaft“, „Englisch - Globalität und Transkulturalität“, „Mathematik“, „Kreativität und Gestaltung“, „Gesundheit und Soziales“, und „Natur und Technik“ (ebd.). Daraus sind als Pflichtfächer Deutsch, Englisch und Mathematik sowie ein eigenes Modul zur Berufsorientierung und als Wahlfächer zwei der weiteren oben genannten, beziehungsweise eine weitere Sprache, zu wählen (ERWACHSENENBILDUNG.AT O.J.).

Als zentraler Begriff für den erwachsenengerechten Pflichtschulabschluss hat sich in den letzten Jahren jener der „Kompetenzen“ etabliert (BUNDESMINISTERIUM FÜR 
Unterricht, Kunst UND Kultur 2013, pp. 25 ff. $)^{23}$. Obwohl dafür eine Vielzahl von Definitionen vorliegt und ähnlich zum Integrationsbegriff nicht von einer Einheitlichkeit ausgegangen werden kann, manifestiert sich ein wichtiger Aspekt darin, dass Kompetenzen auch außerhalb des Bildungssektors ,informell“" erworben werden können. Damit können bereits vorhandene Fähigkeiten und Qualifikationen, insbesondere von Geflüchteten und Migrant/inn/en, vermehrt anerkannt werden. Dies ist ein Schritt von einer Defizitorientierung zu einem stärker aufbauenden Ansatz. Es gibt allerdings auch Kritikpunkte, die sich vor allem einerseits auf die vermehrte Quantifizierung und Testung von Kompetenzen beziehen und andererseits den Imperativ der ökonomischen Verwertbarkeit von Wissen und Bildung hinterfragen. Kompetenzen sind demnach

„verhaltensregulierende persönliche Potentiale und Dispositionen, die sich vorwiegend aus der reflektierten Verarbeitung praktischer Erfahrungen entwickeln und jeweils zur Bewältigung verschiedener Anforderungssituationen verwendet werden können. “ (RÜTZEL 2010, p. 3 zit. nach BUNDESMINISTERIUM FÜR UNTERRICHT, KUnST UND KULTUR 2013)

Der Fokus auf die Stärkung von Kompetenzen anstelle der Vermittlung von Wissen ist also besonders im Kontext von Erwachsenenbildung für Asylwerber/innen und Asylberechtigte ein wichtiger Ansatz. Dadurch kann sowohl die Lebensrealität der Zielgruppe mitbedacht werden als auch eine erwachsenengerechte Umsetzung der Bildungsmaßnahmen, die auf den Erfahrungen und informellen Qualifikationen aufbaut, sichergestellt werden. Damit wird betont, dass die Aneignung von Kompetenzen nicht ausschließlich über Bildungsmaßnahmen erfolgt, sondern sich maßgeblich aus Lebenserfahrungen und beruflichen Betätigungen speist, selbst wenn diese nicht durch Zeugnisse oder Zertifikate belegbar sind. Im Folgenden werden zwei unterschiedliche Anbieter von Pflichtschulabschlusskursen für Geflüchtete in Mödling und Wien vorgestellt und anhand der empirischen Beispiele verdeutlicht, welche Themen sowohl für die Trägerorganisationen als auch die Asylwerber/innen und Asylberechtigten von besonderer Bedeutung sind.

\section{BACH Bildungszentrum in Mödling}

Das BACH Bildungszentrum in Mödling ist eine Einrichtung der Diakonie, welche für Geflüchtete und Migrant/inn/en Basisbildungs- sowie Pflichtschulabschlusskurse und muttersprachliche Bildungs- und Berufsberatung anbietet. Für den Pflichtschulabschluss gab es mit Stand Juli 2018 drei parallellaufende Kurse zu jeweils circa 18 Personen. Finanziert wurden diese Angebote vorwiegend durch Förderungen von Landes- und Bundesstellen und aus EU-Mitteln. Zu Beginn fand für die Teilnehmer/ innen ein Einstufungstest statt, um im Anschluss daran den Vorkenntnissen entspre-

$23 \quad$ Dieser Absatz beruht auf einer detaillierten Behandlung der Verwendung des Kompetenzbegriffes im Kontext von Pflichtschulabschlusskursen für Erwachsene aus der „Handreichung zum Pflichtschulabschluss: pädagogisch - didaktische Überlegungen“ (2013) des Bundesministeriums für Unterricht, Kunst und Kultur. 
chend weitgehend homogene Gruppen zu bilden. Aus diesem Grund mussten immer wieder Interessent/inn/en abgelehnt werden, die nicht in die jeweilige Gruppe passten. Die Zielgruppe der Maßnahmen waren Menschen mit Migrations- oder Fluchtbiographie. Diese Personen haben bei allgemeinen Angeboten für Pflichtschulabschlusskurse häufig Schwierigkeiten mit den Anforderungen Schritt zu halten und benötigen ein zusätzliches Betreuungsangebot sowie verstärkte Sprachförderung. Dem wird im BACH Bildungszentrum Rechnung getragen. So gibt es monatliche Gespräche zu den Fortschritten in den einzelnen Gegenständen aber keine sozialpädagogische Betreuung oder Sozialarbeit. Wenn notwendig wird allerdings auf spezialisierte Stellen verwiesen.

Im Gegensatz zu den Basisbildungskursen des Roten Kreuzes wird im BACH Bildungszentrum großer Wert auf einen möglichst homogenen Wissensstand der Teilnehmer/innen gelegt. So werden die Gruppen in den unterschiedlichen Gegenständen nach den Kenntnissen der Schüler/innen im jeweiligen Fachgebiet gebildet. Der Vorteil dieses Systems ist, dass die Schüler/innen besser imstande sind, dem Unterricht $\mathrm{zu}$ folgen und im Optimalfall weder über- noch unterfordert werden. Dies ergibt sich im Vergleich zu den Basisbildungskursen womöglich auch aus der Notwendigkeit einem strengeren Curriculum zu folgen und am Ende eine Prüfung abzulegen, deren Zeugnis zum Besuch von weiterführenden Schulen berechtigt. Außerdem ist immer nur eine Lehrperson anwesend, was einen differenzierten Unterricht schwieriger macht, weil nicht so leicht unterschiedliche Schwierigkeitsgrade in den Aufgabenstellungen umgesetzt werden können.

\section{„Projekt Schule für Alle“ (PROSA) in Wien}

Das „Projekt Schule für Alle“ (PROSA) ist ein weiterer Anbieter für Pflichtschulabschlusskurse für Geflüchtete und Menschen mit Migrationsbiographie. Nach vier Semestern Unterricht können die Teilnehmer/innen als Externist/inn/en die Pflichtschulabschlussprüfung an einer Wiener Tagesschule ablegen. Dabei wird nach einem Modulsystem vorgegangen, sodass einzelne Gegenstände schon abgeschlossen werden können, bevor in allen Fächern das dafür notwendige Wissen erworben ist. In den letzten sechs Jahren vor der Erhebung, welche die Basis dieser Studie bildete, ist die Anzahl der Schüler/innen stark angestiegen, mit Stand Oktober 2018 wurden ungefähr 100 Personen unterrichtet. Zielgruppe sind alle Menschen, die sich im österreichischen Bildungssystem in einer marginalisierten Position befinden oder zu alt sind, um reguläre Tagesschulen zu besuchen. PROSA finanziert sich über Privat- und Firmenspenden, aber auch über Förderungen und Kooperationen mit anderen Organisationen. Im Gegensatz zum BACH Bildungszentrum wird ein Großteil des Unterrichts von Ehrenamtlichen geleistet, die zuvor eine entsprechende Einschulung inklusive der Grundlagen einer rassismuskritischen Bildungsarbeit erhalten. Der Unterricht findet häufig im TeamTeaching statt. Dabei werden auch Absolvent/inn/en eingebunden, die inzwischen den Pflichtschulabschluss nachgeholt haben und sich ehrenamtlich einbringen. Dadurch ergeben sich zusätzliche sprachliche Möglichkeiten, um Aufgaben in den Mutter- 
sprachen der Teilnehmer/innen zu erklären. Ein weiterer Vorteil ist, dass Personen, die sich bereits in Österreich etabliert haben von ihren Erfahrungen und Erfolgen berichten können.

Bei PROSA besteht ein ausgeprägtes Bewusstsein dafür, dass die Teilnehmer/ innen mit vielen unterschiedlichen Herausforderungen zu kämpfen haben, welche sich auf den schulischen Fortschritt auswirken. Ein umfassendes Unterstützungsprogramm wird durch die Organisationsstruktur von PROSA sichergestellt. PROSA ist Teil des Vereins „Vielmehr für Alle!“”, der neben Pflichtschulabschlusskursen auch Wohnraum für Geflüchtete vermittelt, ein Buddy-Projekt betreibt und bei der Orientierung auf dem Arbeitsmarkt unterstützt. Die Bildungstätigkeiten von PROSA finden dabei im „Dreieck von Sozialarbeit, Kursangebot [...] und Inklusionsarbeit/Nachbarschaftsarbeit" (PANZENBÖCK 2017, p. 3) statt. Auch der schulische Betrieb wird um ein Unterstützungsangebot durch Sozialarbeit ergänzt, da ansonsten ein gelingender Unterricht kaum möglich wäre (vgl. Interview TRAINERIN PFLICHTSCHULABSCHLUSSKURS PROSA 2018). Die Notwendigkeit dieser vielfältigen Angebotspalette ergibt sich neben der spezifischen Situation von Asylwerber/inne/n in Österreich durch die weit verbreitete Betroffenheit von Rassismus und struktureller Diskriminierung von Geflüchteten:

,,[Es gibt] einfach eine massive Ungleichheit, die da herrscht und eine massive Ungerechtigkeit, der sie da ausgesetzt sind, die sie spüren. Und auch Alltagsrassismus zum Beispiel. Also sprechen wir auch viel darüber, wie geht man damit um, wenn man im Bus auf einmal einfach beschimpft wird, weil man halt Bus fährt. “ (Interview Trainerin PfLichtschulabschlusskurs PROSA 2018)

\subsection{Spezifische Anforderungen an Bildungsmaßnahmen für Geflüchtete}

Von den oben vorgestellten Anbietern berichteten alle von einer starken Nachfrage an Bildungsangeboten für Geflüchtete, welche die vorhandenen Kapazitäten bei weitem überstiegen. Zunächst wurde in den Jahren 2016 und 2017 das Kursangebot entsprechend ausgebaut, blieb allerdings immer noch hinter der Anzahl der benötigten Plätze zurück (Steiner, EgGer-Steiner \& Baumegger 2018, pp. 19 ff.). Obwohl die Anzahl der Asylanträge in den vergangenen zwei bis drei Jahren stark rückläufig war, sind im selben Umfang auch die angebotenen Maßnahmen weniger geworden und Fördersummen zurückgegangen ${ }^{24}$. Damit hat sich nichts daran geändert, dass die verfügbaren Kursplätze nicht genügen, um den Bedarf zu decken. Der entscheidende Punkt in diesem Zusammenhang ist die Schaffung einer ausreichenden Anzahl an zielgruppenad-

$24 \quad$ Während in den Jahren 2015 und 2016 rund 88.000 beziehungsweise 42.000 Personen in Österreich um Asyl angesucht haben, waren es 2017 nur mehr etwa 25.000, 201814.000 und 201913.000 (BUNDESMINISTERIUM FÜR INNERES 2019, p. 4). Von rückläufigen Maßnahmen oder geringeren Förderungen berichteten sowohl die Trainer/innen für Basisbildung in Tulln als auch bei PROSA in den jeweiligen Interviews. 
äquaten und finanzierbaren Angeboten im Bildungsbereich. Dazu gehört vor allem die Anerkennung der spezifischen Situation und der relevanten Problemlagen von Geflüchteten in Österreich. Dies umfasst auf der einen Seite entsprechende Begleitmaßnahmen, um ausreichend Unterstützung zu bieten, damit sich Asylwerber/innen und Asylberechtigte verstärkt auf den Fortschritt in den jeweiligen Bildungsmaßnahmen konzentrieren können. Auf der anderen Seite müssen flächendeckende Angebote geschaffen werden, welche für die Zielgruppe erreichbar und mit deren finanziellen Möglichkeiten im Einklang stehen. Wie bereits beschrieben, werden seitens der Anbieter von Bildungsmaßnahmen verschiedene Strategien verfolgt, um mit den spezifischen Anforderungen von Geflüchteten als Zielgruppe umzugehen. Diese unterscheiden sich nach den Rahmenbedingungen der Kurse, den Inhalten, der organisatorischen Ausgestaltung und anderen Faktoren. Trotzdem sind die Herausforderungen, welche die Kursanbieter zu meistern haben, einander oft recht ähnlich. Im Folgenden wird auf die wichtigsten dieser Besonderheiten genauer eingegangen und weitere Möglichkeiten aufgezeigt, um darauf zu reagieren.

\subsubsection{Zur Bedeutung von Vorkenntnissen und Bildungserfahrungen}

Als eine der zentralen Herausforderungen bei der Integration von Geflüchteten im Bildungssektor werden die sehr unterschiedlichen und teilweise recht geringen Bildungserfahrungen von Asylwerber/inne/n und Asylberechtigten genannt (BIFFL 2017). Statistische Erhebungen weisen dabei vor allem auf große Unterschiede innerhalb dieser Gruppe hin. So wiesen etwas mehr als die Hälfte der Geflüchteten aus Syrien einen sekundären oder postsekundären Abschluss auf, während dies bei nur knapp einem Drittel der Afghan/inn/en der Fall war (BUBER-EnNSER et al. 2016, p. 10). Es gilt allerdings anzumerken, dass diese Statistiken zwar ein nützliches Hilfsmittel zur Erhebung des Bedarfs und der konkreten Ausarbeitung von Bildungsmaßnahmen darstellen, allerdings über individuelle Fälle nur wenig auszusagen vermögen. Trotzdem sind derartige Erhebungen zur Sicherstellung eines ausreichenden Angebotes von Bildungsmaßnahmen von großer Bedeutung. Mindestens ebenso wichtig wie die Nationalität erscheinen allerdings Faktoren wie beispielsweise das Alter oder Geschlecht von Geflüchteten. Dazu kommt, dass die Lebenssituationen in den Krisengebieten vor der Flucht durch unterschiedliche Einschränkungen geprägt waren. So war in vielen Fällen infolge von Kriegshandlungen kein Zugang zu Bildung möglich oder Frauen und Mädchen waren in Gebieten, die von (religiösen) Fundamentalisten beherrscht wurden, vom Schulbesuch ausgeschlossen.

Wie bereits erwähnt gibt es je nach Anbieter und Inhalten verschiedene Strategien im Umgang mit heterogenen Gruppen im Rahmen eines Sprachkurses oder einer Bildungsmaßnahme. Diese Zugangsweisen unterscheiden sich zum Teil je nach Art des Angebots und der Rahmenbedingungen der Kurse. Anhand der gewählten empirischen Beispiele lässt sich tendenziell folgendes Muster feststellen: Je höher die Anforderungen einer Bildungsmaßnahme an die Teilnehmer/innen sind, desto mehr 
Wert wird darauf gelegt, dass möglichst homogene Gruppen geschaffen werden. So betonen sowohl PROSA als auch das BACH Bildungszentrum die Notwendigkeit eines möglichst einheitlichen Wissenstandes zu Beginn einer Maßnahme. Zur Vermittlung von fachlichen Inhalten sind außerdem ausreichende Deutschkenntnisse unerlässlich, ansonsten macht eine Teilnahme am Unterricht keinen Sinn (vgl. Interview LEITER BACH Bildungszentrum 2018). Bei der Basisbildung, wo weniger die Aneignung von Wissen als die Befähigung zur selbstständigen Alltagsführung im Mittelpunkt steht, kann es unter Umständen sinnvoll sein Gruppen gemeinsam zu unterrichten, damit gegenseitige Lerneffekte ermöglicht werden können. Nötig dafür ist allerdings ein entsprechender Rahmen, etwa mit zwei Trainer/inne/n, so wie das beim Kurs vom Roten Kreuz in Tulln gehandhabt wurde. Auch im ehrenamtlichen Deutschkurs in St. Andrä-Wördern und im Mostviertel wurden die Teilnehmer/innen in Gruppen eingeteilt, diese waren jedoch recht klein und wurden jeweils von einer Lehrperson betreut. Eine weitere Möglichkeit besteht darin, Gruppen im Bedarfsfall zu trennen, wie dies eine Lehrerin des Pflichtschulabschlusskurses des WIFI berichtete:

„,Wir machen es auch so, wenn jetzt die Gruppe homogener ist, dann machen wir mehr als gemeinsame Gruppe. Ist die Gruppe sehr unterschiedlich, in dem was die Vorkenntnisse sind, dann teilen wir die Gruppe halt oft. Oder wenn es jetzt zwei, drei sind, die komplett irgendwie nicht den Anschluss finden, dass man mal eine Zeit mit zwei, drei arbeitet und schaut, dass man mit denen so lange geht, bis sie den Anschluss wieder finden. Da sind wir, weil keine Schule, sehr flexibel. Und können total individuell arbeiten. Das ist sehr positiv. " (Interview LEHRERIN DES PflichtschulabschlussKuRses WIFI 2018)

Die hier angesprochene Flexibilität ist sicher eine entscheidende Qualität für die Organisation und Durchführung von Bildungsangeboten für Geflüchtete. Somit gibt es keinen Zugang, der im Allgemeinen empfohlen werden könnte, sondern verschiedene Konzepte, die sich in unterschiedlichen Situationen bewährt haben. Im Zusammenhang mit dem Umgang mit heterogenen Vorkenntnissen von Geflüchteten stellt sich außerdem die Frage nach der Bedeutung dieses Faktors für den Bildungserfolg in Österreich. Die Daten aus den Interviews zeigten, dass es auch hier nicht sinnvoll ist zu generalisieren:

„, Und das haben wir eigentlich auch bei den Infotagen, dass wir dann schon immer die Teilnehmer befragen: Was war vorher? Weil, das spielt sehr stark auch in den Unterricht bei uns. Weil wenn der sagt, er hat acht Jahre Schule in seinem eigenen Land, dann wissen wir, der weiß wie man lernt. Auch wenn er jetzt nicht Deutsch unbedingt so gut kann. Es gibt aber Teilnehmer, die waren noch nie in irgendeiner Unterrichtsmaßnahme, da kann es aber sein, dass die mit Eltern auf der Flucht waren, wo der Vater sehr gut unterrichtet hat. Also es gibt nichts, wo man irgendwie etwas Generelles sagen kann. " (Interview LeHrERIN Des PFLICHTSCHULABSCHLUSSKURSES WIFI 2018)

Der Begriff der Bildungserfahrungen umfasst also in diesem Kontext zwei unterschiedliche Bedeutungsebenen: Auf der einen Seite vorhandenes Wissen in unter- 
schiedlichen Bereichen wie beispielsweise Mathematikkenntnisse oder den Umgang mit Computerprogrammen, andererseits beziehen sich Bildungserfahrungen auch auf die Kompetenzen sich neues Wissen anzueignen und über eine individuelle Lernstrategie zu verfügen. Normalerweise wird davon ausgegangen, dass sich im Rahmen einer schulischen Laufbahn diese beiden Ebenen parallel entwickeln. Allerdings besteht die Möglichkeit, sich Wissen außerhalb eines schulischen Kontextes anzueignen, und für Fortschritte in einzelnen Wissensgebieten sind die vorhandenen Grundlagen nicht unbedingt maßgeblich. Es ist jedenfalls nicht so, dass für Personen, die länger in der Schule waren, die Integration im Bildungssektor in Österreich zwangsläufig einfacher ist:

„Nein, also ich könnt' jetzt nicht klar sagen, dass diejenigen, die länger in der Schule waren, auch schneller Deutsch lernen. Es gibt sicher einen Fall, der kann super Englisch, der hat schnell Deutsch gelernt. [...]. Genauso hab'ich einen der außer Paschto ursprünglich keine andere Sprache konnte, [der] hat dann in der Unterkunft Dari gelernt und lernt jetzt Deutsch und da geht's grad auch wahnsinnig schnell weiter. [...]. Ja, dann ist ein anderer dabei, der war acht Jahre in der Schule und tut sich trotzdem voll schwer, also das ist unterschiedlich." (Interview Trainerin BasisbiLdung Rotes Kreuz Tulln 2018)

Das hängt höchstwahrscheinlich auch damit zusammen, dass weitere individuelle Faktoren wie beispielsweise mögliche traumatisierende Erfahrungen vor und während der Flucht, die Art und Weise wie Asylwerber/innen mit der belastenden Situation des offenen Verfahrens umgehen, aber auch die Auffassungsgabe und Motivationsfähigkeit sehr unterschiedliche sind. Was allerdings auffällt ist, dass Geflüchtete mit einem Universitätsabschluss eher gewohnt sind zu lernen und sich daher doch leichter tun, sich neues Wissen anzueignen (vgl. Interview Trainerin Pflichtschulabschlusskurs PROSA 2018). Besondere Herausforderungen ergeben sich, wenn Asylwerber/innen oder Asylberechtigte Analphabet/inn/en sind und erst in Österreich und auf Deutsch Lesen und Schreiben lernen (Interview KoORDinatorin eHRENAMTlicher DeUtschKuRs St. ANDRÄ-WÖRDERn 2018). Es kommt also doch stark darauf an, welche Möglichkeiten Geflüchtete nach ihrer Ankunft in Österreich vorfinden. So kann es, beispielsweise wie im Fall von St. Andrä-Wördern, einen Deutschkurs direkt in der Nähe der Unterkunft geben, während manchmal Quartiere recht isoliert liegen und der Zugang zu Bildungsmaßnahmen nicht vorhanden ist. Viele der Unterschiede entstehen somit erst nach der Ankunft in Österreich (vgl. Interview Leiter BACH BiLdungszentrum 2018), was im allgemeinen Diskurs zur Integration von Geflüchteten im Bildungssektor zu wenig berücksichtigt wird. Hier spielen wieder die verschiedenen Komponenten von Integration wie Gesundheit, soziale Kontakte oder die Wohnsituation eine wichtige Rolle.

So resultieren aus den Bildungserfahrungen im Herkunftsland, den Erlebnissen vor und während der Flucht und den Bedingungen nach der Ankunft in Österreich spezifische Rahmenbedingungen, welche die Verwirklichungschancen von Asylwerber/ inne/n und Asylberechtigten beeinflussen. Es handelt sich hierbei also um eine komplexe 
Gemengelage, die sich durch die Verbindung aus vorhandenem inhaltlichem Wissen, Kenntnissen zur Aneignung neuer Kompetenzen, den Rahmenbedingungen in Österreich und individuellen Faktoren zusammensetzt.

\subsubsection{Traumatische Erlebnisse und die Situation von Asylwerber/inne/n in Österreich}

Von beträchtlicher Relevanz sind belastende Erfahrungen vor und während der Flucht, die sowohl Geflüchtete persönlich als auch deren Familienangehörige und Freunde betreffen können. Diese äußern sich beispielsweise in Konzentrationsschwächen oder posttraumatischen Belastungsstörungen, wie mehrere Mitarbeiter/innen von Organisationen, die Bildungsmaßnahmen für diese Zielgruppe anbieten, berichten:

„Das heißt, das sind einfach extreme psychische Bedingungen plus Vortraumatisierungen aus ihren Heimatländern, zu wenig psychologische Unterstützung in Österreich. Viele sind allein hier [...], oft sehr junge Männer und Frauen, die quasi vollkommen auf sich selbst gestellt sind und die natürlich auch mitbekommen, dass ihre Familien im Heimatland weiter den Bedingungen dort ausgesetzt sind. “ (Interview Trainerin PFLICHTSChUlabschlussKurs PROSA 2018)

Hier zeigt sich einerseits, dass in Österreich häufig keine entsprechenden Rahmenbedingungen zur Aufarbeitung dieser psychischen Belastungen geboten werden. Dies kann unter anderem auf Sprachbarrieren und eine geringe Bereitschaft Unterstützung von Psycholog/inn/en anzunehmen, aber vor allem auch auf ein zu geringes Angebot in diesem Bereich zurückgeführt werden (vgl. Hemayat - BetreuUngSzentrum Für FOLTER- UND KRIEGSÜBERLEBENDE 2013). Andererseits ist mit der Ankunft in Österreich der Prozess des Ankommens noch lange nicht abgeschlossen, worauf beispielsweise in einer Publikation von Kohlbacher und Six-Hohenbalken mit dem Titel „Die lange Dauer der Flucht" aus dem Jahr 2019 hingewiesen wird. An dieser Stelle werden einmal mehr die Besonderheiten der Integration von Asylwerber/inne/n und Asylberechtigten im Bildungssektor deutlich. So berichtete eine der Trainer/innen für Basisbildung in Tulln von den Unterschieden zwischen der Arbeit mit Migrant/inn/en und Geflüchteten:

„Also zum Beispiel Personen, die einschlafen aber nicht jetzt, weil ihnen so fad ist, sondern wo man irgendwie das Gefühl hat, da stimmt was nicht. [...] Da ist irgendwie der Rhythmus verlorengegangen, also das ist sicher ein Thema bei den Teilnehmern. Und auch wenn das Thema Flucht irgendwie aufkommt, also dass das sicher einfach belastende Erfahrungen sind, die man bei anderen Migranten halt einfach nicht berücksichtigen muss. " (Interview MitarbeITERIN MigrationsabTeILung Rotes Kreuz Tulln 2018)

Konzentrationsschwierigkeiten sind in diesem Zusammenhang recht weit verbreitet und unter anderem darauf zurückzuführen, dass Geflüchtete mit vielen unterschiedlichen Schwierigkeiten zu kämpfen haben, die einen großen Teil ihrer Energie und Aufmerksamkeit in Anspruch nehmen. Wie bereits angesprochen sind dabei jedoch 
nicht nur Geschehnisse im Herkunftsland oder auf der Flucht von Bedeutung, sondern auch die Situation, in der sich Asylwerber/innen in Österreich befinden. Zentral ist hier die Unsicherheit während des Asylverfahrens, das Warten auf den ungewissen Ausgang und die allgegenwärtige Gefahr einer Abschiebung als Folge eines negativen Asylbescheids.

„, Genau und das Belastende merkt man halt vor allem dann, wenn irgendjemand aus der Gruppe wieder einen negativen Bescheid bekommen hat. [...] Auch wenn jemand umziehen muss, [...] dass Quartiere geschlossen werden und dann wieder die Unsicherheit da ist, wohin kommen wir oder find 'ich rechtzeitig eine Wohnung, um umzuziehen? So Dinge merkt man auch im Kurs. " (Interview TraINERIN BASISBILDUNG Rotes KReUZ TUlLn 2018)

Der Alltag vieler Asylwerber/inne/n ist von einer Zeit des bangen Wartens und Hoffens auf einen positiven Ausgang des Verfahrens geprägt. Für viele stellt diese Zeit eine große psychische Herausforderung dar, was besonders für den Besuch von Bildungsmaßnahmen relevant ist:

„Das ist so eine Zeit, die so geprägt ist von Lethargie und von schon fast ein bisschen Depressionen, weil einfach - also die Wartezeit im Asylverfahren ja mehrere Jahre teilweise sein kann. [...] Das sind junge Menschen, Großteils junge Männer, die total motiviert sind, wenn sie nach Österreich kommen, und die lässt man irgendwie untätig zu Hause herumsitzen [...]. " (Interview SozIALARBEITERIN DiAKONIE - MOBILER FLÜCHTLINGSDIENST 2018)

Die Ungewissheit, welche vielen Asylwerber/inne/n während des Verfahrens zu schaffen macht, wirkt sich allerdings nicht nur psychisch aus. Auch die Motivation Deutsch zu lernen oder an Kursen teilzunehmen kann wegen fehlender Perspektiven mit der Zeit sinken.

\subsubsection{Thematisierung von Fluchterfahrungen in Kursmaßnahmen}

Die Situation von Asylberechtigten unterscheidet sich in diesem Bereich wesentlich von jener von Asylwerber/inne/n. Nach dem positiven Abschluss des Asylverfahrens ist zumindest für die nächste Zeit der Aufenthalt in Österreich gesichert ${ }^{25}$. Allerdings ist damit auch der verpflichtende Auszug aus den Grundversorgungsquartieren innerhalb von vier Monaten und die Organisation einer eigenen Wohnung verbunden. Gleichzeitig beginnt die Suche nach einem Arbeitsplatz, welche häufig mit vielschichtigen weiteren Herausforderungen einhergeht, auf welche an dieser Stelle nicht näher eingegangen

${ }_{25}$ Wenn Geflüchtete subsidiären Schutz anstelle von Asyl erhalten, erfolgt dies auf der Grundlage, dass bei einer Abschiebung ins Herkunftsland aufgrund der allgemeinen Sicherheitslage vor Ort eine Gefahr für Leib und Leben bestehen würde. Subsidiärer Schutz wird vorerst für ein Jahr verliehen, Verlängerungen um jeweils zwei Jahre sind jedoch möglich (OESTERREICH.GV.AT). Seit 2016 wird Asyl in Österreich (rückwirkend mit dem Stichtag 25.11.2015) nur mehr auf Zeit vergeben. Dies bedeutet zuerst auf drei Jahre beschränkt und nach einer Überprüfung unbefristet (KLINGE 2017, p. 3). 
werden kann (siehe dazu beispielsweise LiCHTENWAGNER 2019; NECHANSKy 2019). Der positive Abschluss des Asylverfahrens eröffnet also eine Reihe an neuen Möglichkeiten, die Verwirklichungschancen von Geflüchteten bleiben dabei allerdings häufig hinter den eigenen Erwartungen zurück. Weiterhin besteht in manchen Fällen zusätzlich die Sorge um Familienangehörige, die in den Krisengebieten zurückgeblieben sind, welche die Geflüchteten auf der Suche nach Sicherheit und einem Neuanfang verlassen haben. Die Herausforderungen, welche sich bei der Integration im Bildungssektor stellen, lassen sich mit dem Erfahrungsbericht einer Trainerin für Basisbildung bei PROSA sehr gut zusammenfassen:

„Also der Druck und die Unsicherheit, die da auf den Schultern dieser Personen lasten, ist unvorstellbar und ist unerträglich und wir haben sämtliche posttraumatische Belastungsstörungen, die man so kennt: Schlafprobleme, Konzentrationsprobleme. Wobei auch bei Asylberechtigten es dann so ist, dass es vielleicht zumindest nicht mehr die eigene Person betrifft, aber halt dann vielleicht die Familienmitglieder noch betrifft und so weiter. [...] Diese Dauer des Asylverfahrens ist eine Extrembelastung für alle Beteiligten. Ja, [...] also ich muss wirklich sagen, ich befrage meine Schülerinnen und Schüler nie nach ihrem Herkunftsland oder nach ihrer Geschichte oder so, aber natürlich passiert es manchmal, dass man Geschichten hört und was diese Leute erleben und durchmachen. Das ist für mich ein Wunder, dass diese Leute es jeden Morgen schaffen aufzustehen und in die Schule zu kommen. " (Interview Trainerin Pflichtschulabschlusskurs PROSA 2018)

Offensichtlich ist, dass es für viele Geflüchtete einer Unterstützung im Umgang mit diesen Belastungen bedarf. Eine Möglichkeit dazu, die immer wieder in Gesprächen erwähnt wurde, bestand darin mit Ehrenamtlichen oder im Rahmen eines Deutschkurses oder Basisbildungskurses darüber zu sprechen. Dies konnte für einzelne Asylwerber/ innen und Asylberechtigte durchaus Erleichterung mit sich bringen, barg aber auch Gefahren in sich: So konnte die Thematisierung von traumatisierenden Erlebnissen für die Ehrenamtlichen selbst eine große Belastung darstellen (vgl. IzQuIERDo et al. 2018). Auf dieses Problem kann durch gegenseitigen Austausch der Trainer/innen oder externe Supervision reagiert werden. Solche Erzählungen sollten jedenfalls nicht seitens der Lehrpersonen aktiv gefördert werden. Dies nicht zuletzt deshalb, um einer möglichen Retraumatisierung vorzubeugen. Es ist aber auch nicht sinnvoll, weil darunter die Vermittlung von Kursinhalten leiden kann, wie ein Lernbegleiter der Basisbildung bei „Bildung hat Wert" berichtete:

„,Viele haben natürlich schlimme Erlebnisse. Manche kommen dann und wollen das auch in die Gruppe bringen, wollen das [...] zur Sprache bringen. Das ist eine sehr schwierige Sache. Früher habe ich dem manchmal Raum gegeben. [...] Nur, ich bin heute so weit, dass, [...] wenn ich spüre, das geht jetzt in so eine Richtung, dass ich in der Regel irgendwie versuche das Steuer rumzureißen und von dem sofort wegzukommen. [...] [Weil] was dann oft passiert ist so eine Dynamik, dass 
andere sich einklinken [...] und dann - man kann dann nichts mehr in Richtung Lernen machen. “ (Interview DAZ-LERnBEGLEITER BASISBILDUNG „BILDUNG HAT WERT" 2018)

Die Sinnhaftigkeit, einem diesbezüglichen Mitteilungsbedürfnis der Geflüchteten nachzukommen, kann somit eher in niederschwelligeren Angeboten gegeben sein. Es gilt allerdings eine Balance zu finden, um sich selbst und die anderen Teilnehmer/innen psychisch nicht zu überfordern und gleichzeitig den eigentlichen Zweck der Kursmaßnahme nicht aus den Augen zu verlieren. Für Trainer/innen ist es außerdem wichtig, aus Selbstschutz eine gewisse Distanz zu wahren:

„Ich kann denen nicht helfen und ich bin keine Psychologin und ich will mich ehrlich auch nicht irgendwie damit beschäftigen, weil, es zieht dann mich auch runter. Ich kann nicht ganz so in persönliche Sachen reintauchen, weil dann komme ich nicht raus selbst. Weil dann zieht es mich runter und das ist nicht gut, glaube ich. Für meine Psyche wäre das auch nicht gut, ja. " (Interview DAZ-TraINERIN MENTOR 2018)

Im Zweifelsfall ist es ob dieses sensiblen Bereiches sinnvoll, professionelle psychologische Unterstützung anzufordern. In Bildungsmaßnahmen, die stärker auf die Vermittlung von Inhalten ausgerichtet sind, ist es für den Lernfortschritt oft kontraproduktiv, wenn individuelle Probleme oder Fluchterfahrungen zur Sprache kommen. Es zeigt sich also, dass diese Angebote zum Teil unter anderen Rahmenbedingungen durchgeführt werden und andere Ziele und Vorgaben haben als niederschwelligere Maßnahmen. Daher kann diesen Themen in weiterführenden Bildungsangeboten häufig weniger Raum gegeben werden. Die langen Wartezeiten im Asylverfahren und die Untätigkeit, unter der die Asylwerber/innen zu leiden haben, kann auch dazu führen, dass sich eine gewisse Frustration über die Gesamtsituation aufstaut. In manchen Fällen entlädt sich diese dann in den Kursmaßnahmen, woraus eine schwierige Dynamik resultieren kann, da sich die meisten Teilnehmer/innen in einer ähnlichen Situation befinden (vgl. Interview DAZ-LeRnBegleIter BASISBILdUng „BILDUNG HAT WERT“ 2018).

Hier bietet sich ein Verweis auf entsprechende, darauf spezialisierte Stellen an. Als zusätzliche Begleitmaßnahme, um auf die spezifische Situation von Geflüchteten einzugehen, hat sich ergänzende Sozialarbeit - wie im Fall von PROSA - bewährt. Die psychologischen Belastungen von Asylwerber/inne/n und Asylberechtigten sind in vielen Fällen sehr hoch, insbesondere in der Zeit während des Asylverfahrens. Das betrifft auch die Anbieter von Bildungsmaßnahmen, und zwar auf vielfache Weise. Einerseits ist die Belastbarkeit gegenüber Leistungsdruck im Vergleich zu anderen Zielgruppen eingeschränkt, worauf in angemessener Weise Rücksicht genommen werden soll. Auf der anderen Seite kann durch persönlichen Kontakt und emotionale Verbindungen das Leid, welches Geflüchtete erlebt haben, für Lehrpersonen zu hohen Belastungen führen. Daher ist es wichtig, die notwendigen Begleitangebote umzusetzen, um Unterstützung zu bieten, aber auch Überforderungen der Trainer/innen durch Supervision und ähnliche Maßnahmen vorzubeugen. 


\subsubsection{Individuelle Faktoren im Zusammenhang mit der Integration im Bildungssektor}

Zusätzlich zu den bereits genannten Schwierigkeiten spielen die Familiensituation und das Geschlecht von Geflüchteten bei der Integration im Bildungssektor ebenfalls eine wichtige Rolle. Für Familien, die mit ihren Kindern geflohen sind, bestehen außerdem Betreuungspflichten, die die Möglichkeit Bildungsmaßnahmen zu besuchen zum Teil stark einschränken. Insbesondere Frauen, die zumeist für Haushaltstätigkeiten verantwortlich sind (vgl. Interview SchULLEITERIN VS ST. ANDRÄ-WÖRdERN 2018), benötigen in diesem Bereich Unterstützung, beispielsweise durch Angebote, welche auch Kinderbetreuung abdecken. Umgekehrt kommt es vor, dass Kinder infolge ihres Schulbesuchs bessere Deutschkenntnisse als die Eltern aufweisen und in verschiedenen Situationen als Übersetzer/innen tätig werden (vgl. Interview ASYLBERECHTIGTER Syrien St. ANDRÄ-WÖRdERn 2018). In diesen Dynamiken und der Veränderung von Rollenbildern, zum Beispiel wenn Männer aufgrund von Arbeitslosigkeit oder Arbeitsverbot während des Asylverfahrens kein ausreichendes Einkommen erwirtschaften können, um die Familie zu versorgen (vgl. Tošı́́, KRONER \& BINDER 2009, pp. 120 f.), besteht ein erhebliches Potenzial von psychischen Belastungen:

„Die Schülerinnen und Schüler, die das Glück haben, dass ihre Eltern mitkommen konnten hierher, das sind dann einfach Eltern, die ihre ganze Energie dafür aufgebracht haben, die Familie in Sicherheit zu bringen. Und dann sind die hier, sitzen in einer Wohnung, haben keinen Zugang zu einem Deutschkurs, haben keinen Zugang zu irgendwas, keinen Zugang zu Arbeit. Das heißt die Kids regeln das alles, die Jungen regeln das alles und die Alten sitzen zu Hause. Und da ist dann der Weg in die Depression quasi vorgeschrieben. Einfach weil keine gesellschaftliche Teilhabe [gegeben ist]. " (Interview Trainerin PFLICHTSCHULABSCHLUSSKURS PROSA 2018)

Mehrere Trägerorganisationen von Bildungsangeboten für Geflüchtete berichten außerdem von Schwierigkeiten, Asylwerberinnen zu erreichen (vgl. STEINER, EGGERSteiner \& BAumegger 2018, p. 32) 26. Dies liegt einerseits daran, dass diese häufiger in privaten Unterkünften untergebracht sind. Dadurch ist die Kooperation schwieriger und die Anreise manchmal nicht so leicht zu organisieren. Andererseits haben diese Frauen oft familiäre Verpflichtungen, aber auch VVorbehalte gegenüber dem Besuch von gemischtgeschlechtlichen Gruppen können eine Rolle spielen. Diese Thematik wird insgesamt eher ambivalent eingeschätzt. Positiv kann festgehalten werden, dass Kurse für Frauen und Männer dazu beitragen können, dass ein Miteinander der Geschlechter zunehmend als selbstverständlich erachtet wird. Allerdings benötigen einige geflüchtete Frauen geschützte Räume, in denen es leichter fällt, traumatisierende Erlebnisse zu verarbeiten und diese profitieren dementsprechend von separaten Bildungsangeboten (ebd.).

26 Die genannte Studie bezieht sich auf junge Geflüchtete im Alter von 15 bis 21 Jahren, die beschriebenen Phänomene treffen allerdings (eventuell abgeschwächt) auch auf ältere Personen $\mathrm{zu}$. 
Ein zusätzliches Erschwernis stellt für viele Geflüchtete die Wohnsituation dar. In Wien war ein Großteil der Asylwerber/innen und Asylberechtigten unter prekären Bedingungen untergebracht (AIGNER 2018). So ist das Ende der Unterbringung in Grundversorgungsquartieren in vielen Fällen nicht gleichbedeutend mit einer Verbesserung der Wohnverhältnisse. Da Wohnraum in Wien knapp ist und Menschen mit Fluchterfahrung besonders in diesem Bereich struktureller Diskriminierung ausgesetzt sind, bleibt vielen keine andere Möglichkeit, als mit mehreren anderen Personen in überfüllten und überteuerten Wohnungen unterzukommen. Diese werden häufig von Mitgliedern der ethnischen Community vermietet. Diesbezügliche Kontakte und die Unterstützung durch Ehrenamtliche können aber zu einer entscheidenden Verbesserung der Situation beitragen (ebd.). Einige der Teilnehmer/innen an den Kursen von PROSA waren auch von Obdachlosigkeit betroffen (vgl. Interview TRAINERIN PFLICHTSCHULABSCHLUSSKURS PROSA 2018). In der Gemeinde St. Andrä-Wördern war dies zwar kein Thema, die Mehrbettzimmer in den Unterkünften boten allerdings nur wenige Möglichkeiten, um sich zurückzuziehen und in Ruhe zu lernen (vgl. Interview EHRENAMTLICHE DeUtSCHLEHRERIN ST. ANDRÄ-WöRDERN 2018).

Es lassen sich also vielerlei Gründe nachweisen, weshalb insbesondere für Asylwerber/innen eine Fokussierung auf den Besuch von Kursen schwierig ist. Das Spektrum beinhaltet sowohl traumatische Erlebnisse vor und während der Flucht, die Unsicherheit über den Ausgang des Asylverfahrens als auch die marginalisierte Position in der österreichischen Gesellschaft (vgl. Abschnitt 3.1). Trotzdem ist es der Einschätzung der Autor/inn/en nach sehr wichtig, noch während des Verfahrens Deutschkurse und andere Bildungsmaßnahmen anzubieten. Einerseits dauern die Asylverfahren häufig sehr lange, sodass Integration bereits in diesem Zeitraum beginnen kann. Andererseits haben viele Asylwerber/innen trotz der widrigen Umstände wegen der verordneten Untätigkeit ein ausgeprägtes Bedürfnis nach Tagesstruktur und sinnvollen Tätigkeiten. Die Angebote von Ehrenamtlichen spielen dabei eine zentrale Rolle, da Integrationsmaßnahmen in den Bereichen Basisbildung und Pflichtschulabschluss von offiziellen Stellen häufig erst nach dem Abschluss des Asylverfahrens vorgesehen sind. Die gesellschaftlichen Rahmenbedingungen tragen somit ihren Teil dazu bei, dass auf diesem Gebiet besonderer Handlungsbedarf besteht.

\subsection{Fazit}

Ateş und ReINPRECHT (2017, p. 303) unterscheiden in Bezug auf Basisbildungsangebote für Erwachsene zwischen zwei verschiedenen Zugängen: Einerseits können auf kompensatorische Weise nachholend Defizite von Personen mit Bildungsbedarf behoben werden, andererseits gibt es Maßnahmen, die versuchen, die bereits vorhandenen Kompetenzen anzuerkennen und diese als Ressourcen betrachten, auf denen es aufzubauen gilt. Wie die empirischen Beispiele zeigen, hängen die unterschiedlichen Zugangsweisen recht stark mit der Philosophie der jeweiligen Anbieter von Bildungs- 
angeboten zusammen. Bei PROSA wurde die Einbettung des Bildungssektors in die gesamtgesellschaftliche Realität von Geflüchteten in Österreich betont. Dementsprechend verstanden sich die Pflichtschulabschlusskurse nur als ein Teil des Angebotes des Vereins „Vielmehr für Alle!“‘. Zusätzlich ging die Bildungsarbeit von einem rassismuskritischen Ansatz aus, der Diskriminierung als eine wichtige Ursache der Bildungsbenachteiligung von Asylwerber/inne/n und Asylberechtigten identifizierte. Die Trainer/ innen des Basisbildungskurses in Tulln betonten den Versuch, auf den bereits vorhandenen Kompetenzen der Teilnehmer/innen aufzubauen und trachteten danach, durch das gewählte Format gegenseitige Unterstützung zu fördern. Im BACH-Bildungszentrum wurden den Geflüchteten regelmäßige Gespräche angeboten, um den Austausch zwischen den Lehrenden und den Lernenden zu fördern. So konnte bei Problemen, die durch externe Faktoren verursacht wurden, auf entsprechende kompetente Stellen, etwa im Bereich Sozialarbeit oder psychologische Unterstützung, verwiesen werden. Der ehrenamtliche Deutschkurs in der Gemeinde St. Andrä-Wördern lebte in besonderer Weise vom Austausch zwischen den Lehrer/inne/n und den Teilnehmer/inne/n, der sich nicht ausschließlich auf die Kurssituation beschränkte, sondern auch bei anderen Veranstaltungen gepflegt wurde.

Zusätzlich zu diesen empirischen Beispielen sind aus der Literatur mehrere entscheidende Kriterien für Basisbildungsmaßnahmen für Erwachsene bekannt (АTEŞ \& REINPRECHT 2017, p. 311): Erstens bedürfen Lerninhalte einer lebensweltlichen Relevanz für die Teilnehmer/innen. Das bedeutet, dass Dinge vermittelt werden müssen, die für Geflüchtete zur eigenständigen Bewältigung ihres Alltages in Österreich wichtig sind. Dies betrifft beispielsweise den Umgang mit Behörden oder das Ausfüllen von Erlagscheinen, wie sie im Basisbildungskurs des Roten Kreuzes in Tulln vermittelt wurden. Zweitens ist eine Niederschwelligkeit gefordert, die sich nicht nur auf materielle Aspekte bezieht: Die Angebote für Asylwerber/innen und Asylberechtigte sollten daher kostenlos und in ihrer zeitlichen und räumlichen Dimension an die Zielgruppe angepasst sein. Das beinhaltet zum Beispiel einen kurzfristigen Einstieg zwischen zwei Kursmaßnahmen wie beim ehrenamtlichen Deutschkurs in der Gemeinde St. Andrä-Wördern, aber auch die Erreichbarkeit in der Nähe des Wohnortes durch öffentliche Verkehrsanbindung und eventuell eine Übernahme der Fahrtkosten. Zum Dritten muss sichergestellt werden, dass Geflüchtete zu den notwendigen Informationen über die Angebote gelangen, um daran teilnehmen zu können. Dies funktioniert in vielen Fällen über ehrenamtliche Unterstützer/innen, zusätzlich ist die muttersprachliche Bildungs- und Berufsberatung des BACH-Bildungszentrums diesbezüglich eine gute Möglichkeit. Auch die Sozialarbeiter/innen des mobilen Flüchtlingsdienstes der Diakonie leisten in diesem Bereich für das Mostviertel wichtige Informationsarbeit. Oft reicht es jedoch nicht aus, wenn diese Informationen vorhanden sind. Es muss zusätzlich sichergestellt werden, dass Asylwerber/innen und Asylberechtigte damit erreicht werden. Außerdem müssen die Informationen in einer Form aufbereitet werden, welche die Wahrscheinlichkeit erhöht, dass die entsprechenden Bildungsangebote dann auch wahrgenommen werden. Viertens fußt Lernen insbesondere in diesem sensiblen Bereich auf einer Vertrauens- 
beziehung, die aufgrund häufiger negativer Erfahrungen mit Diskriminierung große Bedeutung hat. Dieses Vertrauen kann aufgebaut werden, indem der Situation, in der sich Asylwerber/innen und Asylberechtigte befinden, Rechnung getragen wird. Dazu braucht es Verständnis, wenn Dinge nicht auf Anhieb auf die Art und Weise umgesetzt werden können, wie das wünschenswert wäre. Eine weitere Option ist es, den Raum für Themen zu öffnen, die Geflüchtete beschäftigen und nach Maßgabe der Möglichkeiten, wie beim Deutschkurs in St. Andrä-Wördern, darauf einzugehen. Persönliche Beziehungen, die über die Rollen als Wissensvermittler/innen und Lernende hinausgehen, stellen in diesem Zusammenhang eine zusätzliche Qualität vieler ehrenamtlicher Angebote dar. Schließlich beruht das Lernen fünftens wesentlich auf Reziprozität im Sinne eines voneinander Lernens, dass zwischen den Teilnehmer/innen gefördert werden kann, wie etwa beim Basisbildungskurs des Roten Kreuzes in Tulln. Dieser Lernprozess kann und soll aber auch die Lehrenden miteinbeziehen, deren Horizont sich durch den regelmäßigen Kontakt mit Menschen unterschiedlicher Kulturen und Erfahrungen bei einer entsprechenden Bereitschaft, sich darauf einzulassen, fast zwangsläufig erweitert.

Asylwerber/innen und Asylberechtigte als Zielgruppe befinden sich darüber hinaus in einer spezifischen Situation. So sind für die Integration von Geflüchteten im Bildungssektor sowohl deren gesellschaftliche Positionierung als marginalisierte Gruppe in Österreich als auch persönliche Faktoren von Bedeutung. Diese Aspekte sind natürlich individuell unterschiedlich stark ausgeprägt, der Umgang damit hängt mit den persönlichen Ressourcen einzelner Geflüchteter sowie der gebotenen Unterstützung zusammen. Dabei spielen soziale Netze, Beziehungen zu ehrenamtlichen Unterstützer/ inne/n oder die Verfügbarkeit von Integrationsmaßnahmen ebenfalls eine wichtige Rolle. Es gibt somit einige Schwierigkeiten, die Asylwerber/innen im Allgemeinen betreffen, wie beispielsweise die gesellschaftlichen Rahmenbedingungen ihres Aufenthalts in Österreich oder die Unsicherheit bis zum Abschluss des Asylverfahrens. Dazu kommen individuelle Faktoren, die ebenfalls beträchtliche Auswirkungen haben. So ist das Alter wichtig, wenn es um die Integration in den Arbeitsmarkt oder in angepasste Bildungsmaßnahmen geht. Personen über 50 Jahre haben dabei ohnehin keinen leichten Stand, wenn eine adäquate Beschäftigung gefunden werden soll. Das Geschlecht stellt ebenfalls eine zentrale Kategorie dar, da gesellschaftliche Rollenbilder Vorstellungen der Erwerbstätigkeit von Frauen, Ungleichbehandlung und Sexismus die Lebensrealität mitbestimmen. Zusätzlich hat die Familiensituation bedeutsame Auswirkungen. Kinderbetreuungspflichten können die zeitlichen Ressourcen für Deutschkurse, Arbeit oder Bildungsangebote einschränken. Geflüchtete stellen demnach eine sehr heterogene Gruppe dar, die sich nicht nur in Bezug auf die Herkunftsländer unterscheidet. Neben den bereits genannten Faktoren spielen auch berufliche Qualifikationen, Bildungserfahrungen, mögliche Traumatisierungen sowie die Erlebnisse im Herkunftsland und auf der Flucht eine Rolle. Der Umgang mit diesen unterschiedlichen Gegebenheiten ist zusätzlich von Person zu Person verschieden, vor allem was traumatisierende Erfahrungen anbelangt. Diese haben nicht auf alle Menschen dieselben Auswirkungen. Während manche selbst lebensbedrohliche Ereignisse einigermaßen gut verkraften 
können, haben andere unter vergleichsweise weniger dramatischen Begebenheiten lange zu leiden (vgl. EhrenamtLicher Unterstützer St. ANDrä-Wördern 2018).

Der Bildungssektor ist einer der zentralen Bereiche, in dem entscheidende Kompetenzen zur Führung eines selbstbestimmten Lebens angeeignet werden können. Dies betrifft einerseits Deutschkenntnisse, welche sowohl für die Teilhabe am Arbeitsmarkt als auch zur alltäglichen Verständigung wichtig sind. Andererseits geht es darum, sich in Österreich zurechtzufinden und Alltagssituationen eigenständig bewältigen zu können. Diese Themenfelder werden zwar durch Kursmaßnahmen abgedeckt, vielfach findet aber darüber hinaus im Kontakt mit anderen Menschen die praktische Anwendung und Weiterentwicklung von Kompetenzen statt. In den Schulen und weiteren Bildungsangeboten für Erwachsene sind sowohl die heterogenen Bildungserfahrungen von Geflüchteten als auch die Situation, in welcher sich Asylwerber/innen und Asylberechtigte in Österreich befinden, mit unterschiedlichen Herausforderungen verbunden. Hierbei zeigt sich, dass es keine eindeutigen Lösungen gibt, mit diesen Schwierigkeiten umzugehen, sondern dass erfolgversprechende Ansätze immer auf die spezifische Situation der Gruppe, die jeweiligen Bildungsmaßnahmen und andere wichtige Faktoren abgestimmt werden müssen.

Schließlich ist festzuhalten, dass in vielen Fällen die Nachfrage nach geeigneten Bildungsangeboten vor allem für Asylwerber/innen größer ist als das Angebot, da diese Zielgruppe spezifische Anforderungen stellt. Die empirischen Ergebnisse bestätigen somit den Befund, welchen die NGO „SOS Mitmensch“ den Möglichkeiten von Geflüchteten im österreichischen Bildungssystem ausstellt:

„Die Praxis zeigt, dass der Bildungszugang für nicht mehr schulpflichtige Asylsuchende in Anbetracht oftmals unzureichender und vielfach auch unübersichtlicher Angebote stark von engagierten SozialarbeiterInnen bzw. vom Einsatz von Ehrenamtlichen abhängig ist. Dies gilt für die private Finanzierung von Sprachkursen, für Lernhilfen oder auch für das Suchen und Finden von passenden Kursen. Dort, wo es den BetreuerInnen in Einrichtungen an Ressourcen fehlt, bleiben Asylsuchende, die keinerlei ehrenamtliche Unterstützung erfahren, oftmals auf der Strecke und finden keinen Zugang zu den vorhandenen Angeboten. " (SOS Mitmensch 2019, p. 6)

In diesem Kapitel wurden einige relevante Bildungsmaßnahmen für Asylwerber/ innen und Asylberechtigte vorgestellt, die zentrale Aspekte in diesem Bereich abdecken. Der Großteil davon geht von NGOs, zivilgesellschaftlichen Initiativen und ehrenamtlichen Unterstützer/innen aus. Die österreichische Integrationspolitik hat seit den Jahren 2015 und 2016 noch keine ganzheitliche Strategie gefunden, um die Verwirklichungschancen von Geflüchteten zu verbessern. Dies mag unterschiedliche Gründe haben, wobei mangelnder politischer Wille wahrscheinlich nicht den geringsten unter diesen darstellt. In der Zwischenzeit zeigten die Bemühungen von unterschiedlichen Anbietern von Bildungsmaßnahmen und die Unterstützung von Ehrenamtlichen Wirkung, um zumindest für einige Asylwerber/innen und Asylberechtigte den Weg zu einer nachhaltigen Integration in diesem wichtigen Bereich zu ebnen. 